\title{
SAR for Wearable Antennas with AMC Made using PDMS and Textiles
}

\author{
Muhammad Nazrin Ramli ${ }^{(1)}$, Ping Jack Soh ${ }^{(1,2)}$, Hasliza A. Rahim ${ }^{(3)}$, Mohd Faizal Jamlos ${ }^{(1)}$, Fatin Nabilah Giman ${ }^{(1)}$, Ezzaty \\ Faridah Nor Mohd Hussin ${ }^{(1)}$, Herwansyah Lago ${ }^{(1)}$ and Emmanuel Van Lil ${ }^{(2)}$ \\ (1) Advanced Communication Engineering (ACE) CoE, School of Computer and Communication Engineering, Universiti \\ Malaysia Perlis, Pauh Putra Campus, 02600 Arau, Perlis, MALAYSIA \\ (2) ESAT-TELEMIC Research Div., KU Leuven, Kasteelpark Arenberg 10 Box 2444, 3001 Leuven, BELGIUM \\ (3) Bioelectromagnetics Research Group, School of Computer and Communication Engineering, Universiti Malaysia Perlis, \\ Pauh Putra Campus, 02600 Arau, Perlis, MALAYSIA
}

\begin{abstract}
Besides the radiation and reflection performance of wearable antennas, arguably one of the most important parameters is their Specific Absorption Rate (SAR). This work aims to evaluate SAR for wearable antennas integrated with Artificial Magnetic Conductor (AMC) plane made using different material categories - textiles and a flexible polymer,. Two types of textiles, felt and ShieldIt Super are used to build the first, textile-based antenna, while polydimethylsiloxane (PDMS) and the fluidic metal eutectic gallium indium alloy (EGaIn) are used to build the second, polymer-based antenna. Both materials are chosen due to their flexibility conformity to the human body, thus providing comfort to users. Despite the SAR for both antenna types did not exceed the European regulatory limits of $2 \mathrm{~W} / \mathrm{kg}$ averaged over $10 \mathrm{~g}$ of tissues; there are considerable differences between them.
\end{abstract}

\section{Introduction}

Wireless Body Area Networks (WBAN) devices are foreseen to be capable of changing the human paradigm in applications in body sensing, medicine, health monitoring and emergency rescue [1]. The accessibility between the $2.4 \mathrm{GHz}$ WBAN frequency and the widely used Industrial, Scientific, and Medical (ISM) $(5.8 \mathrm{GHz})$ band [2] requires WBAN devices to be capable of operating in both frequencies using a single hardware. Besides that, a wearable system is envisioned to be conformal, lightweight, miniature in size, low profile, inexpensive, and easy to fabricate to ensure its attractiveness. This requires their components, including radiators to be made from special materials such as textiles and flexible polymers.

Another challenge for such antennas is to ensure that their radiations are not directed towards the human user by placing a reflective surface between the body and the antenna. However, to ensure that the antenna maintains a low profile characteristic, it is essential that the reflector is located as close as possible to the radiator. One of the structures which serves this purpose is metasurfaces such as artificial magnetic conductor (AMC) $[3,4,5]$. This is also to enable its safe operating condition for such wearable devices when operating on body, which is characterized by its Specific Absorption Rate (SAR). It is also known from [6] that these values obtained from commercial electromagnetic solvers such as CST Microwave Studio is closely correlated to measurements using commercial setups.

\section{Materials and Antenna Design}

The first antenna, which is a textile-based antenna, is built using two types of textiles: the conductive ShieldIt and the non-conductive felt. The felt substrate used is $3 \mathrm{~mm}$ thick, with a relative permittivity $\left(\varepsilon_{\mathrm{r}}\right)$ of 1.44 , and loss tangent $(\tan \delta)$ of 0.044 . Meanwhile, ShieldIt Super is 0.17 $\mathrm{mm}$ thick with an estimated conductivity of $1.18 \times 10^{5}$ $\mathrm{S} / \mathrm{m}$. The overall structure for this textile based antenna consists of five layers, three layers of ShieldIt and two substrates based on [7]. It features a textile dipole placed on the top-most layer, followed by a $3 \mathrm{~mm}$ thick felt substrate. Next, a 3 x 3 AMC plane, which unit cells are formed using rectangular patches slotted with diamond shaped slots, is placed on the intermediate layer. This is followed by another $3 \mathrm{~mm}$ thick felt substrate layer before a full ground plane located at the bottom-most layer. The overall structure is sized at $90 \times 90 \times 6.51 \mathrm{~mm}^{3}$.

On the other hand, a second polymer based antenna is built using two main material categories, the nonconductive polydimethylsiloxane and the conductive eutectic gallium indium alloy and copper plate as its conductors. Similarly, each PDMS substrate layer is also $3 \mathrm{~mm}$ thick, featuring a $\varepsilon_{\mathrm{r}}$ of 2.7, and a tan $\delta$ of 0.0134 [8]. Meanwhile, EGaIn is composed of $75.5 \%$ of Gallium and $24.5 \%$ of Indium resulting in an electrical resistivity of about $29.4 \times 10^{-6} \Omega-\mathrm{cm}$. This antenna cross section is similar to the textile-based antenna, except for an additional $1 \mathrm{~mm}$ thick layer on its top layer to keep the fluidic liquid embedded in the antenna. This is followed by a $3 \mathrm{~mm}$ PDMS substrate layer, with a $40 \times 28 \mathrm{~mm}^{2}$ EGaIn radiator embedded $1 \mathrm{~mm}$ into its thickness form the top. Next, another $3 \mathrm{~mm}$ thick PDMS substrate is placed underneath this layer. An AMC plane formed using $3 \times 3$ rectangular patches made from copper plates is embedded $1 \mathrm{~mm}$ into this second PDMS substrate. 
Finally, a full ground plane formed using a $0.035 \mathrm{~mm}$ thick copper foil covers the bottom-most layer [5]. The overall size of this structure is $147 \times 147 \times 6.035 \mathrm{~mm}^{3}$. Both designs were simulated using CST Microwvae Studio (MWS).

\section{Results and Discussion}

The AMC plane for the textile based antenna indicated a reflection phase bandwidth of $227 \mathrm{MHz}$ (from 2.38 to $2.61 \mathrm{GHz}$ ) in the lower band and $831 \mathrm{MHz}$ (from 5.26 to 6.1 GHz) in the upper band. Meanwhile, a smaller bandwidth is exhibited by the PDMS based AMC plane: $159 \mathrm{MHz}$ (from 2.34 to $2.50 \mathrm{GHz}$ ) in the lower band and $596 \mathrm{MHz}(5.61$ to $6.16 \mathrm{GHz})$ in the upper band. For the overall radiator, the textile antenna in the planar state exhibited operation with a bandwidth of $162 \mathrm{MHz}$ and $592 \mathrm{MHz}$ in the $2.45 \mathrm{GHz}$ and $5.8 \mathrm{GHz}$ bands, respectively. Similarly, the planar PDMS antenna also operated from 2.39 to $2.48 \mathrm{GHz}$ (in the lower band) and 5.62 to $5.90 \mathrm{GHz}$ (in the upper band).

Table 1. Summary of the SAR averaged over $10 \mathrm{~g}$ of tissues for both antennas at two different frequencies and conditions.

\begin{tabular}{|c|c|c|c|}
\hline Ant Condition & $\begin{array}{c}\text { Freq } \\
(\mathrm{GHz})\end{array}$ & $\begin{array}{c}\text { SAR for } \\
\text { PDMS Ant } \\
(\mathrm{W} / \mathrm{kg})\end{array}$ & $\begin{array}{c}\text { SAR for } \\
\text { Textile Ant } \\
(\mathrm{W} / \mathrm{kg})\end{array}$ \\
\hline $\begin{array}{l}\text { Bent at } x \text {-axis; } \\
r=40 \mathrm{~mm}\end{array}$ & \multirow{4}{*}{2.45} & 0.014 & 0.2 \\
\hline $\begin{array}{l}\text { Bent at } x \text {-axis; } \\
r=60 \mathrm{~mm}\end{array}$ & & 0.032 & 0.15 \\
\hline $\begin{array}{l}\text { Bent at } y \text {-axis; } \\
r=40 \mathrm{~mm}\end{array}$ & & 0.043 & 0.14 \\
\hline $\begin{array}{l}\text { Bent at } y \text {-axis; } \\
r=60 \mathrm{~mm}\end{array}$ & & 0.044 & 0.088 \\
\hline $\begin{array}{l}\text { Bent at } x \text {-axis; } \\
r=40 \mathrm{~mm}\end{array}$ & \multirow{4}{*}{5.8} & 0.031 & 0.165 \\
\hline $\begin{array}{l}\text { Bent at } x \text {-axis; } \\
r=60 \mathrm{~mm}\end{array}$ & & 0.023 & 0.97 \\
\hline $\begin{array}{l}\text { Bent at } y \text {-axis; } \\
r=40 \mathrm{~mm}\end{array}$ & & 0.043 & 0.11 \\
\hline $\begin{array}{l}\text { Bent at } y \text {-axis; } \\
r=60 \mathrm{~mm}\end{array}$ & & 0.039 & 0.054 \\
\hline
\end{tabular}

Prior to SAR assessments, the antennas were also bended at two axes ( $x$ - and $y$-axis) and two different radii (at $r=$ $40 \mathrm{~mm}$ and $60 \mathrm{~mm}$ ) to evaluate their performance when worn on body. Despite small changes, the antenna operation in both desired bands are maintained with reflection coefficients of lower than $-10 \mathrm{~dB}$. Due to this, common SAR evaluation frequencies are chosen at 2.45 $\mathrm{GHz}$ and $5.8 \mathrm{GHz}$ to evaluate both antennas. The bent antennas at two axes and using two radii are placed 10 $\mathrm{mm}$ from the upper arm of a Hugo human body model available in CST, as seen in Figure 1. A summary of the result presented in Table 1 indicated that both antennas are capable of maintaining SAR levels of less than 2 $\mathrm{W} / \mathrm{kg}$ as required by the European regulatory standards. It can be observed that the SAR values for the textile antenna are slightly lower at the higher $5.8 \mathrm{GHz}$ compared to at $2.45 \mathrm{GHz}$, while similar values are observed for the PDMS antenna, except when bent at $r=$ $40 \mathrm{~mm}$ at the $x$-axis.

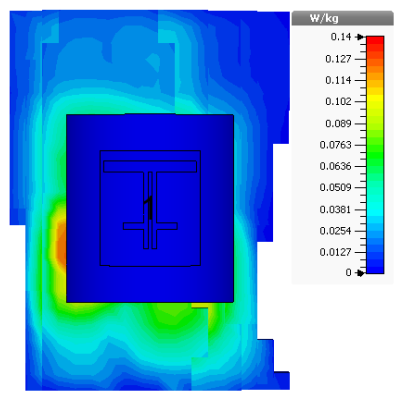

(a)

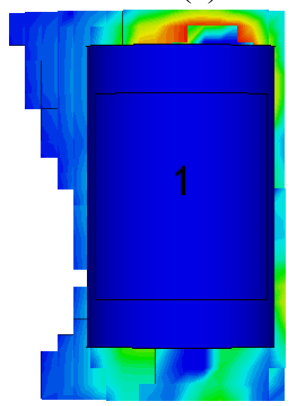

(c)

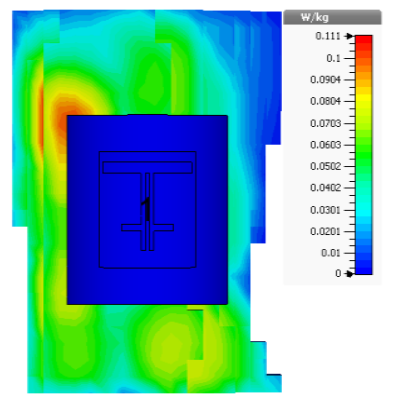

(b)

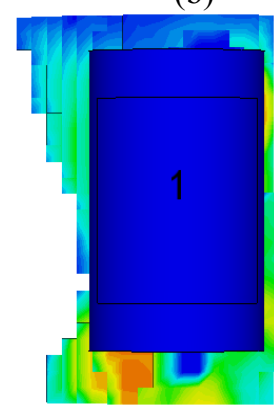

(d)
Figure 1. SAR distribution of antenna bent at the $y$-axis with: (a) textile antenna bent with $r=40 \mathrm{~mm}$ at $2.45 \mathrm{GHz}$; (b) textile antenna bent with $r=40 \mathrm{~mm}$ at $5.8 \mathrm{GHz}$; PDMS antenna bent with $r=40 \mathrm{~mm}$ at $2.45 \mathrm{GHz}$; and PDMS antenna bent with $r=40 \mathrm{~mm}$ at $5.8 \mathrm{GHz}$. [5, 7].

This may be attributed to the contribution of the AMC plane, which shielded the body from potential radiation. Besides that, the larger substrate size and consequently, the larger ground plane is also a contributor to the lower SAR seen in the PDMS antenna.

\section{Conclusion}

This work presents a SAR comparison between two antennas made using different materials. Both antennas featured a radiator, an AMC plane and a full ground plane. This AMC plane is then used as a reflector to reduce back radiation towards potential users when used in a wearable context, while maintaining a planar profile. The first textile-based antenna is built using felt as its substrate and ShieldIt conductive textile as its conductor. Meanwhile, the second polymer-based antenna is built using PDMS as its substrate, a metallic fluid, EGaIn, and copper plates as its conductors. Both antennas are compact in size and featured a dual-band operation. SAR assessments indicated a satisfactory safety level not exceeding the regulatory limit of $2 \mathrm{~W} / \mathrm{kg}$ averaged over $10 \mathrm{~g}$ of tissues. The lower SAR levels for the PDMS based antenna is also due to its inherently larger ground plane caused by the substrate properties. 


\section{Acknowledgements}

This work is supported in part by the MyBrain Scholarships and the Fundamental Research Grant Scheme funded by the Malaysian Ministry of Higher Education (MOHE) (grant no: 9003-00527).

\section{References}

1. Jiang, Z. H, D. E. Brocker, P. E. Sieber, and D. H. Werner, "A compact, low-profile metasurface-enabled antenna for wearable medical body-area network devices," IEEE Transactions on Antennas and Propagation, 62, 8, August 2014, pp. 4021-4030, doi: 10.1109/TAP.2014.2327650

2. J. Tak, S. Woo, J. Kwon, and J. Choi, "Dual-band dualmode patch antenna for on-/off-body WBAN communications," IEEE Antennas Wireless Propagations Letters, 15, June 2016, pp. 348-351, doi: 10.1109/LAWP.2015.2444881

3. S. Yan, P. J. Soh, and G. A. E. Vandenbosch, "LowProfile Dual-Band Textile Antenna With Artificial Magnetic Conductor Plane," IEEE Transactions on Antennas and Propagation, 62, 12, December 2014, pp. 6487-6490, doi:10.1109/TAP.2014.2359194

4. S. Zhu, and R. Langley, "Dual-Band Wearable Textile Antenna on an EBG Substrate," IEEE Transactions on Antennas and Propagation, 57, 4, April 2009, pp. 926935, doi:10.1109/TAP.2009.2014527.

5. M. N. Ramli, P. J. Soh, M. F. Jamlos, H. Lago, N. M. Aziz, and A. A. Al-Hadi, "Dual-band wearable fluidic antenna with metasurface embedded in a PDMS substrate," Applied Physics A, 123, 2, February 2017, pp. 149, doi:10.1007/s00339-017-0754-3.

6. P. J. Soh, G. A. E. Vandenbosch, F. H. Wee, A. van den Bosch, M. Martinez-Vazquez and D. Schreurs, "Specific Absorption Rate (SAR) Evaluation of Textile Antennas," IEEE Antennas and Propagation Magazine, 57, 2, April 2015, pp. 229-240, doi: 10.1109/MAP.2015.2414671

7. F. N. Giman, P. J. Soh, M. F. Jamlos, H. Lago, A. A. Al-Hadi, M. Abdulmalek, and N. Abdulaziz, "Conformal dual-band textile antenna with metasurface for WBAN application," Applied Physics A, 123, 1, December 2016, pp. 32, 2016. doi:10.1007/s00339-016-0626-2.

8. Alqadami, A. S. M., Jamlos, M. F., Soh, P. J., \& Vandenbosch, G., "Assessment of PDMS technology in a MIMO antenna array," IEEE Antennas and Wireless Propagation Letters, 15, December 2015, pp. 1939-1942, doi:10.1109/LAWP.2015.2513960. 